\title{
Ovarian, Fallopian Tube, and Primary Peritoneal Carcinoma pT2 TNM Finding v8
}

National Cancer Institute

\section{Source}

National Cancer Institute. Ovarian, Fallopian Tube, and Primary Peritoneal Carcinoma pT2

TNM Finding v8. NCI Thesaurus. Code C139949.

Ovarian, fallopian tube, or primary peritoneal carcinoma with tumor involving one or both ovaries or fallopian tubes with pelvic extension below pelvic brim or primary peritoneal cancer. (from AJCC 8th Ed.) 